\title{
REFLEXIONES SOBRE LAS IDEAS DE NICOLÁS ORESME
}

\author{
Jorge Alejandro Ramírez Cruz
}

IES Joan Miró. Departamento de Lógica, Historia y Filosofía de la Ciencia de la UNED

\section{RESUMEN}

Este artículo pretende llamar la atención sobre algunas ideas desarrolladas en la Edad Media por Nicolás Oresme (1320-1382) con relación al tratamiento matemático de las cualidades. Se muestra cómo algunos problemas relativos a la interpretación de sus trabajos siguen vigentes aún en nuestros días.

PALABRAS CLAVE: Cualidades, Nicolás Oresme, Edad Media.

\section{REFLECTIONS ON NICHOLAS OREME’S IDEAS}

\section{ABSTRACT}

This paper wants to call attention to some ideas developed in the Middle Ages by Nicole Oresme (1320-1382) in regard to the mathematical treatment of qualities. It is shown how some problems regarding the interpretation of his works are in force even nowadays.

KEY WORDS: Qualities, Nicholas Oresme, Middle Ages.

\section{LA BAJA EDAD MEDIA Y LA MATEMATIZACIÓN DE LA FÍSICA}

Uno de los aspectos relevantes que caracterizaron a la revolución científica del siglo XVII, fue el empleo de las matemáticas en la descripción de los fenómenos físicos. Galileo establece la cinemática de los movimientos de los cuerpos en el vacío y, posteriormente y sobre sus hombros y los de Kepler, entre otros, Newton ve mucho más allá y establece las leyes correctas de la dinámica, que, salvo en el contexto del microcosmos o de velocidades del orden de la velocidad de la luz, gobiernan aún con eficacia el mundo que nos rodea, y la ley de gravitación universal, estableciendo un punto de inflexión en la historia, no sólo de la ciencia, sino del pensamiento humano. Las mareas 
de la ciencia medieval, impulsadas sobre todo por la autoridad de Aristóteles, rompen así en sus últimas playas, luego de dominar la cosmovisón culta de la realidad por centurias. Pero ni mucho menos debe pensarse que estos esfuerzos prenewtonianos fueron estériles. La física de Aristóteles, bien vista y sin complejos, constituyó una razonable filosofía de la naturaleza muy de acuerdo con el sentido común, y capaz de dar explicación, de acuerdo con sus postulados, a muchos fenómenos ${ }^{1}$. Incluso bajo sus postulados se abonó el camino para nuevos planteamientos, tales como los que surgieron entre los siglos XII y XIV sobre el tratamiento las cualidades. El proceso de matematización de los fenómenos físicos, que, como hemos dicho, constituye una piedra angular de la revolución científica, no comienza en el siglo XVII, sino mucho tiempo antes. Como expresa con acierto Crombie ${ }^{2}$ :

«Uno de los cambios más importantes que facilitó el empleo creciente de la Matemática en la Física fue el introducido por la teoría de que todas las diferencias reales podían ser reducidas a diferencias en la categoría de la cantidad; que, por ejemplo, la intensidad de una cualidad, como la del calor, podía medirse exactamente de la misma manera como podía serlo la magnitud de una cantidad. Este cambio es el que distinguió principalmente la física matemática del siglo XVII de la física cualitativa de Aristóteles. Fue comenzado por los escoláticos de la última parte de la edad media».

En efecto, según una tradición que se remonta a Aristóteles y que está presente en las discusiones de los filósofos naturales y de los teólogos de la edad media, la cantidad y la cualidad constituyen dos categorías diferentes. La cantidad se concibe como una entidad constituida por partes homogéneas. El volumen, por ejemplo, que puede pensarse como formado por la agregación de volúmenes más pequeños. Un carácter distinto tienen las cualidades de los cuerpos, tales como la blancura de su superficie o el calor. Resultaba claro que no se podía conferir a éstas las propiedades de aditividad de las cantidades. Sin embargo, se reconocía que podía haber diferentes intensidades o grados para una cualidad dada. La fuerte discusión relativa a la posible matematización del tratamiento de las intensidades, parece arrancar con el análisis hecho por los escolásticos, con Tomás de Aquino como figura más destacada a la cabeza, de una de las sentencias del teólogo Pedro Lombardo (ca. 10901160), quien en su primer Libro de Sentencias señala:

1 Sambursky, S. (1956), The Physical World of the Greeks, Londres, Routledge \& Kegan Paul.

2 Crombie, A.C. (1959), Historia de la Ciencia: De San Agustín a Galileo/2, Siglos XIII-XVII, versión de José Bernia, Madrid, Alianza, 2000 (or.: Oxford University Press, 1959), pp. 82-83. 
«En el hombre, la caridad aumenta o disminuye, y, en épocas diferentes, es más o menos intensa».

Esta frase, aparentemente inocente y sin mayores implicaciones, genera entre los escolásticos una serie de disquisiciones sobre el modo de aumento o de disminución de las intensidades de las cualidades, que constituye uno de los principales objetos de análisis durante la baja edad media. Con sus idas y venidas, puede decirse que durante los siglos XII, XIII y XIV se gestó una evolución en el pensamiento medieval que llevó al tratamiento de la variación de la intensidad de una cualidad similar al existente para la variación de una cantidad, es decir, a pensar en la adición de partes o grados de intensidad. Ricardo de Middleton (†entre 1300 y 1309) es uno de los precursores de esta concepción, que se aleja de las tesis peripatéticas. En esta línea, Francisco de Meyronnes (ca. 1325) establece, como comenta Duhem, «una completa paridad entre el crecimiento de una magnitud continua y la operación por la cual una cualidad, tal como el calor o la caridad, se torna más intensa». Leemos ${ }^{3}$ :

«Al igual que un aumento de extensión sólo puede hacerse por medio de partes extensivas, un aumento de intensidad, tal como un incremento de caridad, sólo puede hacerse por medio de partes intensivas; pero tales partes son grados; el aumento de la caridad se hace por lo tanto por medio de grados»».

En este trabajo se abordan tres aspectos relativos al tratamiento de las cualidades realizado por Nicolás de Oresme que han llevado a diferentes interpretaciones por parte de los historiadores. El primero se refiere a la concepción del continuo empleada por el autor y a sus posibles inconsistencias con el tratamiento peripatético del continuo de uso en la época; el siguiente, el más controvertido, a su verdadero papel en la contribución a la representación geométrica de las intensidades y de la cantidad de una cualidad lineal, que llevaron a Pierre Duhem a señalarlo como el inventor de la geometría analítica. El último, relativo a la interpretación oresmiana de la cantidad de velocidad, tomada ésta como cualidad lineal en el tiempo.

2. LA REPRESENTACIÓN DE LAS CUALIDADES EN ORESME. LOS PROBLEMAS DE INTERPRETACIÓN

Nicolás de Oresme profundizó como nunca antes en la representación de las intensidades de una cualidad. Las interpretaciones que se han hecho de sus

3 Duhem, P. (1936-1956), Le système du Monde, Volumen VII, París, p. 512. 
escritos no son, sin embargo, coincidentes. En lo que sigue intentaremos presentar algunas de sus ideas y señalar y criticar algunas de sus interpretaciones.

Antes de 1371, Oresme desarrolla sus ideas en el Tractatus de configuratione potentiarum et mensurarum difformitattum. Allí generaliza la idea de representar una cualidad puntual por medio de un segmento, a la de representar una cualidad lineal por medio de una superficie, una cualidad superficial por medio de un volumen, e incluso a concebir la idea de una cuarta dimensión espacial para poder representar una cualidad de volumen.

En I.iii del Tractatus, Sobre la longitud de las cualidades, luego de discurrir sobre los nombres que le asignará, Oresme, respetando la tradición, opta por llamar longitud (longitudo) o extensión (extensio) a la línea recta considerada sobre o en el seno del cuerpo afectada por una cierta cualidad, y reserva el nombre de latitud (latitudo) o intensidad (intensio), para designar el grado que adopta la cualidad en un punto dado de la extensión. Según Maier"

«Lo nuevo de esta idea, por contraposición a los métodos ya desarrollados antes de Oresme, de representar una intensidad por una línea, consiste en la extensión de este método a magnitudes de más dimensiones».

En I.iv del Tractatus, Sobre la cantidad de las cualidades, leemos ${ }^{5}$ :

«La cantidad de una cualidad lineal debe ser imaginada por medio de una superficie cuya longitud o base es una línea trazada sobre un tal sujeto, como lo dice el capítulo precedente, y cuya latitud o altitud está representada por una línea elevada perpendicularmente sobre dicha base, de la forma indicada en el segundo capítulo. Por cualidad lineal entiendo la cualidad de una recta del sujeto dotado de la cualidad. Es evidente que la cantidad de una cualidad tal se puede imaginar con la ayuda de una superficie de este tipo, dado que se puede dar una superficie igual en longitud o en extensión a la cualidad, y semejante en altitud a la intensidad de esta cualidad, como se verá claramente más adelante».

En este punto aparece una controversia cuyo origen puede ser más historiográfico que histórico. Según Maier6:

«La introducción de la quantitas qualitatis no es otra cosa que una lógica extensión del principio de línea de intensidad de las cualidades puntuales a las

4 MAIER, A. (1952a), An der Grenze von Scholastik und Naturwissenschaft, Roma, Edizioni di Storia e Letteratura (2. Auflage), p. 291.

5 ORESME, N. (1988), «Tractatus de Configurationibus Qualitatum et Motuum (extraits)», en Nicolas Oresme, Tradition et innovation chez un intellectuel du XIV siècle. Trad. P. Souffrin et J.P. Weiss; ed. por P. Souffrin \& A.Ph. Segonds, París, Les Belles Lettres, p. 137.

6 MAIER (1952a), p. 293. 
cualidades extensas. Se procede de la siguiente manera - si nos limitamos al caso de la cualidad lineal-: sobre la línea que constituye el portador de la cualidad o sujeto, se debe representar en cada punto la línea de intensidad correspondiente, que representa la cantidad de la cualidad en el punto considerado. El conjunto de esas líneas representa la cantidad de la cualidad lineal. $\mathrm{Y}$ ese conjunto de líneas, tal como se admite tácitamente, es una superficie que se eleva sobre la línea del sujeto y cuya altura está determinada por las líneas de intensidad en cada punto aislado, representando su figura el carácter particular de la distribución de intensidad. Análogamente se procede para la cantidad de una cualidad superficial y, al menos teóricamente, de volumen».

Pero es en una nota anexa a este pasaje donde Maier deja ver su crítica a esta concepción ${ }^{7}$ :

«Oresme sabía perfectamente, como la mayoría de sus contemporáneos, que ninguna magnitudo finita puede obtenerse a través de un gran número de indivisibilia; que, en sentido estricto, una línea no se compone de infinitos puntos, ni una superficie de infinitas líneas, etc., pero, en la construcción de sus configuraciones, pasa de puntillas sobre esta dificultad».

La cuestión que plantea Maier es la de que, según la óptica aristotélica, tal como puede leerse en su Física, un continuo no está compuesto por indivisibles $^{8}$ aunque sea infinitamente divisible en partes divisibles. El punto es esencial, porque el mismo Oresme expresa su conformidad con esta idea, cuando en I. $i$ del Tractatus señala9:

«Por otra parte, la intensidad expresa también la idea de que una cosa es «más esto» o «más aquello», por ejemplo, «más blanca»o «más rápida». Esta intensidad, o más precisamente intensidad en un punto, es divisible de una sola forma e infinitamente, como un continuo. No se la puede representar más adecuadamente, por lo tanto, que bajo la forma del continuo que es originalmente divisible y de una sola forma, es decir, por una recta».

La observación que plantea Maier dejaría de ser atendible, en el sentido de revelar una incongruencia en el pensamiento oresmiano, si se considera que del mismo modo en que éste no dice que la recta representativa de una cualidad puntual esté constituida por puntos, sino sólo que es infinitamente divisible, tampoco dice (ver la cita más arriba) que la superficie representativa de una

7 MAIER (1952a), p. 293, nota 13.

8 Aristóteles (1995), Física, Madrid, Gredos, pp. 336-340 y FurLeY, D.J. (1982), «The Greek Commentators' Treatment of Aristotle's Theory of the Continuous», en Infinity and Continuity in Ancient and Medieval Thought. Editado por Kretzmann N., Ítaca y Londres, Cornell University Press, pp. 17-35.

9 ORESME (1988), p. 135. 
cualidad lineal esté formada por el agregado de las líneas de intensidad correspondientes a cada punto, tal como, con Maier, también ha interpretado Clagett $^{10}$, con lo que sí incurriría en dicha inconsistencia. Probablemente ambos autores son partidarios de esta concepción de las ideas de Oresme a partir del análisis que éste hace, en esa misma parte del Tractatus, cuando, refiriéndose a la extensión de esta idea a más dimensiones, dice ${ }^{11}$ :

«Y como en un sólido cualquiera hay un número infinito de superficies iguales para las que se representa la cualidad por un sólido, no es impropio sino necesario imaginar un sólido allí donde otro sólido o cualquier otro sólido está simultáneamente representado»».

Sin embargo, no veo que de aquí se desprenda el que Oresme considere a la superficie representativa de una cualidad lineal como agregado de líneas, ya que, en el comentario recién citado, no establece de modo palpable que el conjunto o agregado de estas superficies constituyan el sólido. Más bien considero que ésta es una interpretación anacrónica llevada por la conjunción del texto oresmiano citado y una posterior exposición galileana, análoga, pero no igual, tal como señala Sellés ${ }^{12}$, que parte de una concepción distinta del concepto de continuo que no entraba en los planteamientos oresmianos.

Otro punto de disputa aparece en la interpretación del tratamiento hecho por Oresme de las cualidades lineales, donde Duhem cree ver al inventor de la geometría analítica ${ }^{13}$.

En I.xi del Tractatus leemos ${ }^{14}$ :

«Así, se podría decir que una cualidad es uniforme si es de igual intensidad en todas sus partes, o que una cualidad uniformemente disforme es tal que para tres puntos cualesquiera, la relación entre la distancia del primero al segundo y la distancia del segundo al tercero es igual a la relación entre el exceso de la intensidad en el primer punto con respecto al segundo y el exceso de la del segundo con respecto al tercero, si se ha llamado primero al punto en el que la intensidad es la mayor».

10 Clagett, M. (1974), «The Configuration of Qualities and Motions, including a Geometric Prof. of the Mean Speed Theorem», en A Source Book in Medieval Science, Cambridge ( Mass.), Harvard University Press, p. 244.

11 ORESME (1988), p. 137.

12 SELLÉs, M. (2001), «La Teoría de Indivisibles de Galileo y su Geometrización del Movimiento», en Largo Campo di Filosofare, Eurosymposium Galileo 2001. Editado por Montesinos J. y Solís C. La Orotava, Fundación Canaria Orotava de Historia de la Ciencia, pp. 445-456.

13 DUHEM (1936-1956), pp. 534-550.

14 ORESME (1988), p. 139. 


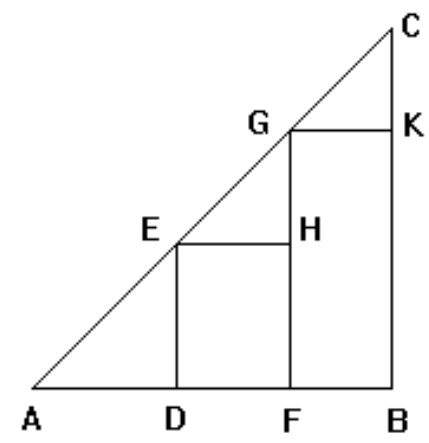

Figura 1 .

«Consideremos en principio una cualidad uniforme acabada en grado nulo, que es simbolizada o representada por el triángulo $\mathrm{ABC}$. Una vez trazadas las rectas perpendiculares $\mathrm{BC}, \mathrm{FG}$ y DE, sea HE paralela a DF y, análogamente, GK paralela a $\mathrm{FB}$. Se tienen así dos triángulos $\mathrm{CKG}$ y GHE, que son equiángulos. De acuerdo con la proposición VI.4 de Euclides, la relación entre GK y EH es igual a la relación entre el exceso CK y el exceso GH. Y como GK es igual a FB y también $\mathrm{EH}$ es igual a DF, la en relación entre FB y DF de las distancias entre los tres puntos de la base es igual a la relación entre CK y GH entre los excesos de las alturas que son proporcionales a las intensidades en los mismos puntos. Dado que la cualidad de la recta $\mathrm{AB}$ es tal que la relación entre las intensidades de los puntos de la recta es igual a la relación entre las rectas trazadas perpendicularmente a los mismos puntos, la proposición es claramente evidente».

Pero la intención de Oresme no es la de describir algebraicamente una relación entre los puntos involucrados que dé lugar a la ecuación de una recta. En palabras de Souffrin ${ }^{15}$ :

«Incluso si se transcribe el texto de I.xi en simbología literal en la forma

$$
\left(x_{3}-x_{2}\right) /\left(x_{2}-x_{1}\right)=\left(h_{3}-h_{2}\right) /\left(h_{2}-h_{1}\right)
$$

con Duhem, se puede impugnar su interpretación como ecuación de la rec-ta: los tres puntos considerados son tratados de la misma manera y el concepto

15 Souffrin, P.; WeIss J.P. (1988), «Le Traité des Configurations des Qualités et des Mouvements de Nicole Oresme, Remarques sur quelques problèmes d'interprétation et de traduction», en Tradition et Innovation chez un Intellectuel du XIV Siècle: Nicole Oresme, París, Les Belles-Lettres, pp. 125-126. 
de variable está totalmente ausente. Pero nos parece más importante remarcar el hecho de que esta trascripción no es fiel al texto. Las relaciones de las que trata Oresme no son relaciones entre diferencias de segmentos, sino relaciones entre segmentos [...]. La relación descrita por Oresme no corresponde a ocho magnitudes, sino a cuatro: es una simple proporción en el sentido entonces clásico del Libro V de los Elementos de Euclides».

Maier es también contundente en la refutación de la interpretación de Duhem ${ }^{16}$ :

«Oresme no ha introducido en absoluto sus longitudo y latitudo como coordenadas. Él no tiene para nada la intención de determinar con su ayuda la situación de un punto en un sistema de referencia, indicando su longitudo y latitudo. $\mathrm{Su}$ longitudo no es una abscisa en sentido moderno, es, como ya hemos dicho, la extensión total del sujeto considerado[...]. Oresme quiere, en resumidas cuentas, construir figuras geométricas, y no curvas en un sistema de referencia».

El último aspecto de los trabajos de Oresme que consideraremos aquí se refiere a su conocida prueba geométrica del teorema del grado medio para las cualidades, que, sin embargo, no está exenta de diferentes interpretaciones. En III.vii del Tractatus, Sobre la medida de las cualidades y de las velocidades disformes, leemos ${ }^{17}$ :

«Toda cualidad uniformemente disforme tiene la misma magnitud que la cualidad del mismo sujeto, o de un sujeto igual, que fuera uniforme, con el grado del punto medio del sujeto dado; esto, sobreentendiendo que el sujeto es lineal. Si es una superficie, tendría el grado de la línea mediana, y si es un sólido, el grado de la superficie mediana, siempre entendiendo los conceptos de forma análoga.

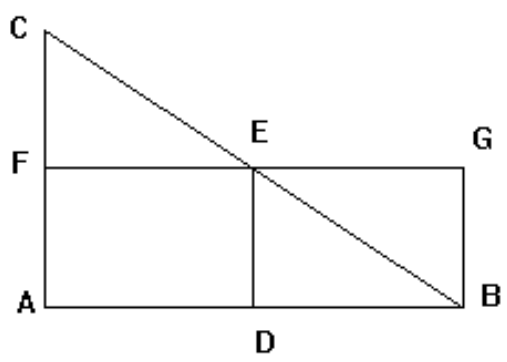

Figura 2.

16 MAIER (1952,a), p. 294.

17 ORESME (1988), pp. 141-142. 
Se lo mostrará en principio para una cualidad lineal. Sea entonces una cualidad representada por el triángulo $\mathrm{ABC}$, uniformemente disforme terminada en grado nulo en el punto B. Sea D el punto medio de la recta del sujeto. El grado o la intensidad de ese punto se representa por la recta DE. La cualidad que sería uniforme sobre todo el sujeto con el grado DE puede representarse por el rectángulo AFGB, de acuerdo con el capítulo diez de la primera parte. Por la Proposición 26 del Libro I de Euclides, se establece que los dos pequeños triángulos EFC y EGB son iguales. Por lo tanto las cualidades así representadas por el triángulo y por el rectángulo son iguales. Y esto era lo que estaba propuesto [...]. Se debe hablar de una velocidad exactamente de la misma forma que de una cualidad lineal, cogiendo como punto medio el instante medio del tiempo que mide una velocidad de ese tipo.

Se ve, por lo tanto, a qué cualidad o a qué velocidad uniforme es igual una cualidad o una velocidad uniformemente disforme».

Para Duhem, considerando como longitudo el tiempo y como cualidad la velocidad, resulta claro que el área, que representa en el pensamiento oresmiano la cantidad de velocidad, se identifica con el espacio recorrido por el cuerpo y coincide dimensionalmente con él, como en la misma línea también argumenta Clagett ${ }^{18}$ :

«La figura en su conjunto, consistente de todas las perpendiculares, representa la distribución completa de intensidades en la cualidad, es decir, la cantidad de la cualidad, o, en el caso del movimiento, la así llamada velocidad total, dimensionalmente equivalente al espacio total recorrido en el tiempo dado».

En este razonamiento se ha identificado al grado de velocidad en un instante - que, según Oresme, tiene la significación general de segmento proporcional a la intensidad de la velocidad, considerada como cualidad, de la cual participa el cuerpo en ese instante, pero sin definición alguna de lo que esto significa o de cómo se cuantifica, del mismo modo que se habla de blancura en un punto o de caridad de una persona en momento dado - con la velocidad instantánea, que, por lo demás, es un concepto muy posterior formalizado por Varignon. En este sentido, merecen señalarse las consideraciones de Anneliese Maier, referidas al concepto mismo de grado de una cualidad y del concepto de grado de velocidad, como una velocidad instantánea medieval, digamos así, no homologable al concepto actual de velocidad instantánea ${ }^{19}$ :

«El punto de partida (Cap.1) es la antigua idea de la linea intensionis, que era corriente especialmente en la literatura médica. Se trata de la representa-

18 Clagett (1974), p. 244.

19 MAIER (1952,a), p. 290. 
ción gráfica de una magnitud intensiva por medio de una línea, de forma tal que a las intensidades mayores les corresponden líneas más largas, tales que las proporciones entre las intensidades y las líneas son iguales. Aquí se debe precisar que estas consideraciones tienen un carácter puramente especulativo, ya que no existe la posibilidad de medir realmente la propia intensidad, ni tampoco existe la posibilidad de comprobar que la relación entre las intensidades es realmente igual a la relación entre las líneas. Por lo demás, éste es un rasgo distintivo de la ciencia natural escolástica, sobre el que ya hemos reparado varias veces: se calcula, antes de que se pueda medir».

De acuerdo con el establecimiento de razones sólo entre magnitudes homogéneas, tal como era de uso en la Edad Media según la tradición griega, establece Maier ${ }^{20}$ :

«[...] la velocidad no se concibe como proporción entre espacio y tiempo.»

$\mathrm{Y}$, más adelante, aludiendo a las consecuencias que habría tenido una definición tal de velocidad, señala ${ }^{21}$ :

«Los escoláticos habrían podido deducir la velocidad instantánea a partir del concepto de velocidad de un movimiento uniforme; pues si la velocidad fuera igual al espacio recorrido en un determinado tiempo, entonces se seguiría consecuentemente que la velocidad instantánea sería igual al espacio (infinitamente pequeño) recorrido en el pequeño lapso de tiempo considerado (Augenblick, en el texto original de Maier), y, de aquí, sólo habría habido un paso para establecer que la velocidad es igual al cociente diferencial entre espacio y tiempo. Está claro que en el siglo XIV no se podía dar ese paso[...]. La solución aceptada por la casi totalidad de los escolásticos tiene una naturaleza distinta; consiste en la introducción de un segundo concepto de velocidad completamente diferente, que es más metafísico que físico y que no tiene ninguna relación con el primero: la velocidad, para decirlo brevemente, es considerada como intensidad del movimiento[...]. El movimiento local es un accidente intrínseco del cuerpo y la velocidad es la intensidad de ese accidente. Puede ser constante, también puede variar, puede experimentar un intendi y remitti como las cualidades, de forma que la intensidad sea diferente en cada instante [...]. Se afirma que un móvil tiene una velocidad de 2, de 4, o en general de $a$, sin que se plantee lo que eso significa, o a qué unidad de medida deben referirse esos números. Y, en virtud de esos datos, se realizan cálculos aparentemente exactos con las cantidades consideradas, que naturalmente tienen poco que ver con la realidad y que carecen de significado físico».

20 MAIER, A. (1952,b), Die Vorläufer Galileis im 14. Jahrhundert, Roma, Edizioni di Storia e Letteratura, p. 120.

21 MAIER (1952b), pp. 121-123. 
Los argumentos de Maier parecen difíciles de rebatir en este punto, y son, además, consistentes con el hecho de que, como ha observado Souffrin ${ }^{22}$, en el texto de Oresme correspondiente al capítulo III.vii, no aparece mención alguna al espacio recorrido por el cuerpo. Sin embargo, la interpretación que hace de esta omisión es bien diferente. La atribuye al hecho de considerar que Oresme se refiere aquí al movimiento en general, entendido en sentido aristotélico general como cambio, y no al movimiento local, que se corresponde, según la física peripatética, con el movimiento de un cuerpo en el espacio. Esta hábil interpretación, que en principio no puede descartarse, no resuelve sin embargo los problemas de fondo que afronta Maier. Souffrin acepta que Oresme «conoce la aplicación de su análisis al caso particular del movimiento en el espacio o 'movimiento local', y se sabe que él adopta en ese caso la acepción usual y singular de 'espacio recorrido' para velocitas totalis».

Ciertamente, en el capítulo III.viii, en el que Oresme muestra con un ejemplo que la cantidad de una cualidad puede tener un valor finito, aunque en algunos tramos de la extensión la intensidad tienda a infinito, leemos ${ }^{23}$ :

«Del mismo modo, si un móvil se desplazara durante la primera parte proporcional de un tiempo dividido de esta manera con una cierta velocidad, dos veces más rápido durante la segunda parte, tres veces más rápido durante la tercera, cuatro veces más rápido durante la cuarta y continuara así infinitamente de forma creciente, la velocidad total sería exactamente cuatro veces la velocidad total de la primera parte, de forma tal que el móvil recorrería, en la totalidad del tiempo, una distancia exactamente igual al cuádruple de la distancia recorrida durante la primera mitad de ese tiempo».

También en las Questiones super geometriam Euclidis de Oresme, lee$\operatorname{mos}^{24}$ :

«Si un cuerpo $a$ se mueve uniformemente durante una hora y $b$ lo hace de forma uniformemente desacelerada en la misma hora, comenzando con un grado [de velocidad] dos veces [el de $a$ ] y terminando en grado nulo, entonces recorrerán distancias iguales, tal como se puede probar fácilmente. Por lo tanto, por la definición de velocidad, se debe conceder que ambos se han movido con la misma velocidad durante la misma hora».

Nótese que en esta referencia lo que se hace realmente es considerar que el cuerpo ha sufrido, en ambos casos, en total, la misma velocidad, en tanto

22 SOUFFrIN-WeISS (1988), p. 127.

23 ORESME (1988), p. 143.

24 DAmerow, P. [et alt] (1992), Exploring the limits of preclassical mechanics, Berlín, Springer, p.18. 
que cantidad de velocidad, ya que las áreas - representativas de sus cantidades respectivas - son, en ambos casos, iguales. De ahí la conclusión de Oresme de que el espacio recorrido final por ambos cuerpos será el mismo. De aquí podemos concluir que las áreas representativas de la cantidad de velocidad son entre sí como los espacios recorridos por los cuerpos, aunque dicha área, siguiendo el análisis de Anneliese Maier, no corresponda dimensionalmente o conceptualmente con el espacio recorrido, como se afirma en la interpretación habitual.

\section{CONSIDERACIONES FINALES}

Podemos concluir que existe una interpretación del área representativa de la cantidad de una cualidad lineal, que no muestra inconsistencias con la concepción aristotélica de Oresme sobre la infinita divisibilidad del continuo sin considerarlo formado por indivisibles, si se piensa que éste considera el área como un todo y no como un agregado de líneas de intensidad.

Por otra parte, la interpretación de Oresme como «creador de la geometría analítica» parece haber sido suficientemente refutada, sin menoscabo de su contribución al proceso de matematización y de visualización geométrica del tratamiento de las cualidades.

Por último, podemos considerar el área, en la representación de la velocidad tomando como longitudo el tiempo, como la medida de la cantidad de velocidad y, de ahí, inferir que áreas iguales corresponden a espacios recorridos iguales, sin que esto implique que el área corresponda dimensionalmente o conceptualmente al espacio recorrido, aunque exista una identificación clara de espacio total recorrido con movimiento total experimentado por el cuerpo, en el sentido de cantidad total de velocidad experimentada por el cuerpo.

Fecha de recepción: 11 de octubre de 2006

Fecha de aceptación: 14 de enero de 2007 